\title{
Highly Ensured QOS Routing in Mobile Ad Hoc Networks Based on Multiple Constraints
}

\author{
D.S. Thenmozhi \\ Lecturer \\ Institute of Road and Transport Technology \\ Erode, Tamilnadu, India.
}

\author{
Dr. R. Lakshmipathi \\ Professor \\ St. Peter's Engineering College, \\ Chennai, Tamilnadu, India.
}

\begin{abstract}
Routing protocols for mobile ad hoc networks have been explored extensively in the past years. Most of the work is aimed at finding a shortest path between the source and the destination, without considering the network performance influencing factors like present network traffic, movements and application requirements etc. In the present world many applications that involve Mobile Ad hoc NETworks (MANETs) contain multimedia data that require Quality of Service (QoS) support for effective transmission. Basically meeting QoS is a mesh of multiple parameters. Many research works so far concentrated QoS routing based on single constraint only. QoS routing based on multiple constraints has been proven to be NP-Complete. Hence, hardly any exact algorithms were proposed for this problem. In this paper a new approach for QoS routing is given based on multiple constraints. In MANETs routing based on Ad hoc On Demand Distance Vector (AODV) protocol provides efficient route establishment between nodes with minimal control overhead and reduced route acquisition latency. In this paper the normal AODV is extended to perform QoS routing based on bandwidth requirement and link stability constraints. Simulation results show that QoS performance of extended AODV is improved in the considerable manner.
\end{abstract}

\section{Keywords}

Quality of Service, bandwidth estimation, contention, link stability, random destination, statistical approach, multiconstraint, simulations.

\section{INTRODUCTION}

Ad hoc wireless network is made up of a group of mobile nodes and all communication is carried out through wireless medium in a distributed fashion without centralized controller. Nodes in MANETs are small radio devices with limited computational capacity and memory. Because of limited radio propagation range, routes are mostly multi-hop. The most desirable advantage of ad hoc networks is their easy and quick deployment. Such an easy deployment would be the most advantageous in a variety of applications ranging from military operations and disaster relief to commercial applications.

Networking mechanisms such as routing protocols for MANETs require high efficiency because of limited resources in a mobile node such as network bandwidth, memory capacity and battery power. Routing in ad hoc networks has to adapt to the unexpected link breakage and topology changes. To discover and maintain the routes in ad hoc networks require more control traffic. This makes the task of performing ad hoc network routing more complex and less efficient. The nature of dynamic changing topology in ad hoc network introduces difficulties in end-to-end route finding.

A lot of work has been made on routing in ad hoc networks: the destination - sequenced distance vector (DSDV) protocol [6], the wireless routing protocol [11], the temporally-ordered routing algorithms [12], the dynamic source routing protocols [13], the associativity based routing protocol [14], and the zone routing protocol (ZRP) [15], etc. These protocols tend to establish a path with least number of hops and achieving a high degree of availability of nodes involved in the active path where the network topology changes quickly. Also, all the previous routing solutions only deal with the best-effort data traffic.

Currently, a lot of applications have been developed for wireless networks, their practical implementation and use in the real world has been limited so far. Many of these applications, such as realtime video and voice are sensitive to the quality of service. Hence focus has been shifted from best-effort services to the provision of better defined QoS in ad hoc networks. In MANETs hard QoS (i.e. guaranteed throughput and delay) is difficult to achieve. Following are the hurdles to achieve hard QoS.

- The dynamic nature of MANETs makes it difficult for hosts to determine information about their local neighbourhood, much less the global status of the network.

- Lack of centralized control, it is very difficult to establish cooperation between neighbouring hosts to determine a transmit schedule for guaranteed packet delivery.

- Signal fading and interferences make the channel more unreliable.

Shared nature of the medium, unstable transmission links in MANETs need additional care at the time of quality of service support.

For most of the QoS required applications basic constraint is throughput [3] i.e. number of bits per second. This is probably because assured throughput is somewhat of a "lowest common denominator" requirement. Not only for text files but also most voice or video applications require some level of guaranteed throughput in addition to other constraints. Hence one of the constraints that are considered in this paper is 'throughput'. Other commonly employed QoS metrics [3] are link stability - the measure of predicted lifetime of a link, link reliability - indicates the chance of a packet or frame being transmitted over a link and correctly decoded at the receiver, end-to-end delay - the measured end to end delay on a path, node buffer space -the 
number of packets in a node's transmission buffer, delay jitter the measured delay variance on a path, packet loss ratio - the number of packets which is not received by the higher layers at the packet's final destination node etc.

Fundamentally route lifetime is based on node battery charge and link stability. Node battery charge [8] is the physical layer metric. The parameter 'link stability' which is the major contributor for the prediction of expected route lifetime, is given due consideration in this paper for QoS routing. Most of the QoS supported routing algorithms are sensitive to the degree of node movements. As nodes move faster the performance in supporting QoS degrades and the control overhead and computational complexity increases nonlinearly. Hence designing the effective routing algorithm is essential. Node movements cause link breakages in MANETs. Thus instead of selecting weak links which may break soon and introduce maintenance overhead one can find path involving stable links i.e. having longer predicted lifetime. By accounting link stability [7] in routing algorithm, the routing overhead can be considerably reduced and the QoS performance can be improved a lot. In our work the Ad hoc On demand Distance Vector routing is extended to find path meeting the application stipulated bandwidth requirement and link stability metrics.

\section{RELATED WORKS}

In MANETs Quality of Service based routing is a relatively new problem. In this section we present an overview of the existing solutions. Cross layer multi constraint QoS routing is proposed in [1]. In this paper Fan proposes multi constraint routing based on MAC delay metric, link reliability and throughput constraints. MAC delay metric is defined as the time between the packets being received by the MAC protocol from the higher layers, and an ACK being received from the destination. He also considered the deferred time when waiting for channel access. Link reliability and throughput constraints are also considered in his work based on their existing definitions and methods of calculations. Fan insisted the fact that the multi constraint QoS routing problem is NP- complete when a combination of additive and multiplicative metrics is considered. He proposed a method for reducing this NP-complete problem to one that can be solved in polynomial time. Advantage of this approach is the simultaneous consideration of several important QoS metrics in path selection. But the QoS state for all paths must be discovered and kept fresh. The mechanism required for this are not discussed in his work. In [7], link stability is considered as a QoS metric. Link stability is majorly dependent on the node movement pattern. This paper presents the Probability Distribution Function (PDF) of link lifetimes under various node movement models. The residual link lifetime is calculated as the area under the PDF for a given mobility model, taken between the links measured lifetime so far and infinity. To find the probability that a link's residual lifetime is greater than a time $t$, the PDF of the link lifetime is integrated between $t+L_{p}$ and infinity, where ' $L_{p}$ ' is the link's past lifetime. This mechanism is combined with AODV for QoS routing. An application may specify a lower limit for acceptable path failure probability $\mathrm{P}_{\mathrm{r} \text {-fail }}$ which is calculated from the delay, delay jitter and packet loss ratio parameters. The value $\mathrm{P}_{\mathrm{r} \text {-fail }}$ is inserted into RREQ packets. Intermediate nodes test that the cumulative failure probability of links up to that point, is not greater than $\mathrm{P}_{\mathrm{r} \text {-fail. }}$. This statistically predicting link lifetime and therefore avoiding links and paths that have a high probability of failure is a very useful mechanism.
To provide quality of service, the network considered must be combinatorial stable [9]. Combinatorial stability means, given a specific time frame, the topology changes occur sufficiently slowly to allow successful propagation of all topology updates as required. Normally QoS can be achieved by coordinating the transmission schedule of packets between nodes. Actually existing approaches are mostly based on local decisions. All are focusing on the packet level and only deal with required resource allocation at individual node point of view. In order to support quality of service, guarantees for end to end flows, path finding approaches need to be combined with suitable admission control strategy. At the time of making admission control decisions, a node considers its local resources simultaneously it must account the resource availability of its contention neighbours because nodes flow may consume their resources through contention.

In [10], the normal route finding method of AODV is improved as Quality of service Enhanced Ad hoc on Demand Distance Vector (QEAODV) routing. In this paper QEAODV establishes a path between the source and the destination meeting the application stipulated throughput requirement. Contention which is the inherent problem in MANET is considered effectively on QEAODV. This extended AODV performs path finding with less overhead by adapting passive approach of listening to the medium. Each node performs admission control based on the details of local bandwidth available and contention-neighbourhood bandwidth available. In this paper admission control involves two phases. During the initial phase only preliminary admission control is done. Full admission control is done only during the second phase. In this approach QEAODV performs better than AODV in terms of throughput and control message overhead. A present trend that we have observed is that many researchers play great emphasis on the session admission (QoS route finding) capability of their protocol, which is admittedly most important but they often neglect the importance of session completion i.e. maintaining the routes and the QoS as long as the application data session requires. Ultimately the session completion is more important from a user perspective than session admission. In this paper session admission is implemented to choose the path which will meet the requested QoS through contention aware routing and will probably last for the requested time period.

The rest of the paper is organized as follows. Section 3 gives major protocol design considerations. Section 4 explains the complete functioning of multi constraint based admission control. Section 5 deals with extended AODV route discovery process. The protocol simulation and results are discussed in section 6 .

\section{DESIGN CONSIDERATIONS}

Some of the metrics commonly used by applications to specify QoS requirements to the routing protocol are minimum required throughput, maximum tolerable delay, maximum tolerable delay jitter, maximum tolerable packet loss ratio. Metrics usually employed by routing protocols for path evaluation and selection to improve all-round QoS are end-to-end delay, node buffer space, energy expended per packet, route lifetime, MAC delay, link $\underline{\text { stability, link reliability etc. }}$

Among the above metrics bandwidth i.e. minimum bits per second requirement of an application is the most wanted metric of most of the real time multimedia based applications. Solutions are suggested by researchers to find path meeting the application stipulated bandwidth requirement and hence admission control 
decision is done based on this. But they have not considered the sustained QoS support based on bandwidth though the link breakages occur. Link stability i.e. the predicted lifetime of a link is the most important criteria determining the assurance of QoS support. Bandwidth and link stability constraints are considered in our work for effective QoS support. Our extended multi constraint based routing by AODV algorithm is designed in such a way that it provides QoS robustness also.

\section{PROTOCOL DESCRIPTION}

Basic AODV [4] is based on flooding the network with Route Request (RREQ) messages. The source node broadcasts a RREQ message with a time-to-live value equal to 1. i.e. a broadcast is limited to one hop neighbourhood. Each RREQ is uniquely identified through a sequence number, so that the first copy of a RREQ received by a node is processed, while duplicated messages are discarded. When a node receives the first copy of a given RREQ it records the address of the node that sent the message. When the first RREQ reaches the desired destination, a Route Reply (RREP) message is generated and sent back to the source node through the recorded reverse path, ensuring a path from the source to the destination. Normally this approach minimizes the number of hops of the chosen path. The basic functionality of our multi constraint routing is much similar to the AODV protocol. Our extended AODV differs from normal AODV in the way the route discovery process is changed to provide quality of service support by performing multi constrained admission control at each node in the network. Similar to AODV, the extended approach also uses the Route Request, Route Reply and Route Error packets for the route discovery and maintenance process, except the Route Request and Route Error packet formats are changed to carry additional information through the network.

The main problem of the MANET comes from the shared nature of the wireless medium in single-channel networks. We focus on ad hoc networks based on single-channel MAC layers like IEEE 802.11 because these single channel protocols are widely available and typically support ad hoc communication. Moreover, these protocols are simple to implement and robust and do not rely on stringent time synchronization that is hard to implement in ad hoc network. The physical characteristics of wireless channels introduce the two challenges. First challenge is available bandwidth estimation at a node, second challenge is estimation of flow bandwidth requirement in a shared medium. In shared wireless medium, when a node starts to transmit a flow, it consumes bandwidth from its contention neighbors. Because each node has a different view of the network, the node cannot decide on its own whether its contention neighbors have sufficient unused bandwidth for the new flow. Also, obtaining contention neighbor information is not easy since a node may consume the bandwidth of contention neighbor but not able to directly communicate with that neighbors if that neighbors are located outside transmission range but inside carrier-sensing range. Multiple nodes on a route may contend for bandwidth at a single location and not know about each other. A node on the route of flow cannot tell how much bandwidth the flow will consume at its contention neighbors.

\subsection{Available Bandwidth Calculation}

The objective of admission control is to determine whether the available resources can meet the requirements of a new flow while maintaining bandwidth levels for existing flows. Each node views a different channel state. The available bandwidth in the network is not a local concept. To tackle this condition, two terms are introduced: local bandwidth available $\left(\mathrm{BW}_{\text {local }}\right)$, contentionneighborhood bandwidth available $\left(\mathrm{BW}_{\mathrm{c} \text {-neigh }}\right)$. Local bandwidth available is the amount of unconsumed bandwidth as observed by a given mode. Contention neighborhood available bandwidth is the maximum amount of bandwidth that a node can use for transmission without affecting the reserved bandwidth of any existing flows in its carrier-sensing range.

In this paper, admission control strategy involves two phases. In the first phase preliminary admission control is done. On receiving RREQ packet a node performs partial admission control. Application flow bandwidth requirement $\left(\mathrm{BW}_{\text {flow }}\right)$ is compared against node's local bandwidth available $\left(\mathrm{BW}_{\text {local }}\right)$ and contention neighbourhood bandwidth available $\left(\mathrm{BW}_{\mathrm{c} \text {-neigh }}\right) . \mathrm{BW}_{\text {flow }}$ is calculated by considering only single node's transmission point of view. Bandwidth requirement of contention neighbours which are also involved in the same active flow are not considered at this stage. During the second phase of admission control known as final admission control node performs full fledged admission control. In this $\mathrm{BW}_{\text {local }}$ and $\mathrm{BW}_{\mathrm{c}-\text { neigh }}$ are compared against actual flow bandwidth requirement $\left(\mathrm{BW}_{\mathrm{a}_{\text {fflow }}}\right) . \mathrm{BW}_{\mathrm{a}_{\text {fflow }}}$ is computed based on the contention count $\left(\mathrm{C}_{\mathrm{ct}}\right)$ of the node.

\subsubsection{Calculation of Local Bandwidth Available (BW $\left.W_{\text {local }}\right)$}

It is the unconsumed bandwidth at a given node. Each node in the MANET can determine its $\mathrm{BW}_{\text {local }}$ by passively listening network activities. In our approach, we use the fraction of channel idle time based on the past history as an indication of local available bandwidth at a node. A node can perceive the channel as either idle or busy. The channel is idle if the node is not in any of the following three states. First, the node is transmitting or receiving a packet. Second, the node receives a RTS or CTS message from another node, which receives channel for a period of time specified in the message. Third, the node senses a busy carrier with signal strength larger than a certain threshold, called the carrier-sensing threshold, but the node cannot interpret the contents of the message. By monitoring the amount of channel

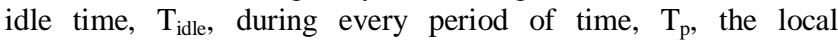
bandwidth available $\mathrm{BW}_{\text {local }}$ of a node can be computed using a weighted average [2] as follows.

$$
\mathrm{BW}_{\text {local }}=\omega \mathrm{BW}_{\text {local }}+(1-\omega)\left(\mathrm{T}_{\text {idle }} / \mathrm{T}_{\mathrm{p}}\right) \mathrm{BW}_{\text {channel }}
$$

Where $\mathrm{BW}_{\text {channel }}$ is the capacity of the channel and weight $\omega \varepsilon$ $[0,1]$. 


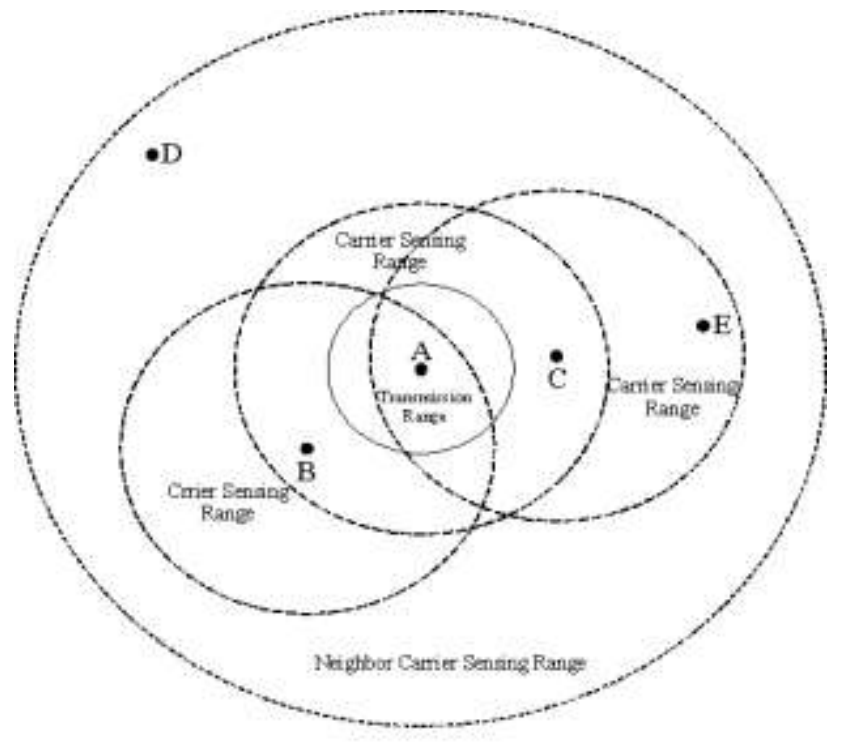

Figure 1. Different sensing ranges of a mobile node

\subsubsection{Calculation of Contention Neighborhood Bandwidth Available $\left(B W_{\text {c-neigh }}\right)$}

Each node perceives the network in a different state. Hence a node's local bandwidth available cannot provide information about its contention neighbors. Since it does not know the amount of $\mathrm{BW}_{\text {local }}$ at other nodes. In our approach, during the normal medium access using IEEE 802.11, a node listens the medium using a threshold value known as contention carrier sensing threshold. In Fig. 1 the inner circle shows the transmission range of node $\mathrm{A}$. Outer circles indicate the carrier sensing range of nodes $\mathrm{B}, \mathrm{A}$ and $\mathrm{C}$ respectively. Normally carrier sensing range is twice the transmission range of a node. Contention carrier sensing threshold refers the range that covers the carrier sensing ranges of all of the sensing node's contention neighbours. Hence it is set to a value much lower than the carrier sensing threshold. When the signal strength of the carrier sensed by a node is smaller than the contention carrier sensing threshold there is no communication in its contention neighborhood and contention neighbors of the node experience idle channels. The amount of time that the channel is in this idle state, denoted as $\mathrm{T}_{\text {idle }}{ }^{\text {contention }}$, for every period of time, $\mathrm{T}_{\mathrm{p}}$, contention neighborhood available bandwidth, $\mathrm{BW}_{\mathrm{c} \text {-neigh }}$ is calculated using the following formula.

$\mathrm{BW}_{\text {c-neigh }} \approx \omega \mathrm{BW} \mathrm{c}_{\text {c-neigh }}+(1-\omega)\left(\mathrm{T}_{\text {idle }}^{\text {contention }} / \mathrm{T}_{\mathrm{p}}\right) \mathrm{BW}_{\text {channel }}$

Where $\mathrm{BW}_{\text {channel }}$ is the capacity of the channel and weight $\omega \varepsilon$ $[0,1]$.

\subsubsection{Calculation of Application's Flow Bandwidth Consumption $\left(B W_{a_{\text {fflow }}}\right)$}

It is required to quantify the bandwidth that a new flow requires so that it can be decided whether the bandwidth available will satisfy the requirements of the flow. Foremost, the application's data rate has to be converted into the corresponding channel bandwidth requirement. As per IEEE 802.11, for every application data packet, the MAC layer performs handshaking. During this RTS, CTS and ACK control packets are involved. Hence each data packet's transmission time is calculated as follows:

$$
\mathrm{T}_{\text {data }}=\mathrm{T}_{\mathrm{rts}}+\mathrm{T}_{\mathrm{cts}}+\mathrm{T}_{\mathrm{ack}}+\mathrm{T}_{\mathrm{difs}}+3 \mathrm{~T}_{\text {sifs }}+(\mathrm{P}+\mathrm{Q}) / \mathrm{BW}_{\text {channel }}
$$

$$
\begin{array}{cl}
\text { Where } \mathrm{T}_{\text {data }} & \text { - transmission time of each data packet } \\
\mathrm{T}_{\text {rts }} & \text { - time for transmitting RTS } \\
\mathrm{T}_{\text {cts }} & \text { - time for transmitting CTS } \\
\mathrm{T}_{\text {ack }} & \text { - time for transmitting ACK } \\
\mathrm{T}_{\text {difs }} & \text { - DCF inter frame space defined in the } \\
& \text { IEEE } 802.11 \text { protocol standard } \\
\mathrm{T}_{\text {sifs }} & \text { - short inter frame space defined in the } \\
& \text { IEEE } 802.11 \text { protocol standard } \\
\mathrm{P} & \text { - size of the data packet } \\
\mathrm{Q} & \text { - IP and MAC packet header length } \\
\mathrm{BW}_{\text {channel }} & \text { - channel capacity }
\end{array}
$$

If at every second, the application generates ' $R$ ' packets with average packet size ' $\mathrm{P}$ ', the corresponding channel bandwidth requirement is computed as follows.

$$
\mathrm{BW}_{\text {flow }}=\mathrm{R} \times \mathrm{T}_{\text {data }} \times \mathrm{BW}_{\text {channel }}
$$

Next factor to be considered is multiple nodes on the route of a new flow may contend for bandwidth at a single location. Every such node needs bandwidth equal to $\mathrm{BW}_{\text {flow }}$. The number of such kind of nodes is known as contention count $\left(\mathrm{C}_{\mathrm{ct}}\right)$. Hence the bandwidth consumption of the flow [2] at this location is expressed as:

$$
\mathrm{BW}_{\mathrm{a}_{-} \text {flow }}=\mathrm{C}_{\mathrm{ct}} \mathrm{x} \quad \mathrm{BW}_{\text {flow }}
$$

\subsection{Link Stability Prediction}

In MANET as nodes move apart network links may break. Some links are subject to have longer lifetime and some links are easily to be broken. Link stability parameter characterizes this feature. Link stability defines the possibility that the link happens to break. Measuring this parameter is not a straight way procedure due to random movement of nodes. However it is observed that in different network environments [7] link stability follows certain statistical properties which enable this parameter to be measured or predicted. The movement pattern of the nodes in the ad hoc networks is a factor that has a considerable impact on network performance. Mobility pattern is important in the sense that the position of nodes at any point of time impacts the network connectivity which is central to the performance of the network.

Node movement in adhoc network is categorised into four patterns. Random Destination, Random Walk, Random Movement and Swarm Movement patterns. In Random destination pattern a node will move towards a destination. Upon arrival, it stays for some time and moves towards another destination. Nodes follow consistent movement in Random Walk model. Swarm Movement means nodes move in group and their relative position will not change much. In Random Movement 
model nodes change each time the direction and speed in a random manner. The links have longest expected lifetime only in the Random Destination pattern. Ad hoc networks set in military and disaster relief situations, their node movements mostly follow random destination pattern. This paper considers link stability property of node movements as per random destination model. This mobility model is found to produce a link lifetime PDF similar to a Rayleigh Distribution[7]. To measure the residual lifetime, the past link lifetime information must be considered.

Its measurement model [5] is described as follows.

$$
\mathrm{P}(\text { residual_lifetime }>\mathrm{t})=\int \operatorname{Rayleigh}(\mathrm{r}) \mathrm{dr}
$$

To find the probability that a link's residual lifetime is greater than a time ' $t$ ', the PDF of the link lifetime is integrated between 't+Lp' and infinity, where 'Lp' is the link's past lifetime.

\section{ROUTE DISCOVERY PROCESS}

During the route discovery process, the source broadcasts route request (RREQ) packet. Application's channel bandwidth requirement $\left(\mathrm{BW}_{\text {flow }}\right)(4)$ is computed by the source and included in the RREQ packet. The application will choose the required $\left(\mathrm{P}_{\mathrm{r}-}\right.$ fail, $\mathrm{T}_{\mathrm{r}}$ ) pair. $\mathrm{P}_{\mathrm{r} \text {-fail }}$ represents the expected route break probability. It is a measure indicating that one of the links along the route may break during the whole flow time. $\mathrm{T}_{\mathrm{r}}$ represents the expected time duration of the flow. Link stability indicator $\left(\mathrm{P}_{\mathrm{r} \text {-fail }}, \mathrm{T}_{\mathrm{r}}\right)$ pair is also inserted in the RREQ packet along with $\mathrm{BW}_{\text {flow }}$ requirement. Each RREQ packet contains the addresses of the source and the destination, the broadcast ID, the last seen sequence number of the destination as well as the source node's sequence number. Broadcast ID is used as an identifier. Sequence numbers are utilized to ensure loop-free and up-to-date routes. A node keeps track of its neighbours by listening to a HELLO message that each node broadcast at set intervals. In addition to the above QoS indicators in the RREQ packet, another field $\mathrm{P}_{\mathrm{a}}$, which represents the accumulated survival probability of all the selected links from the source node to the current node is evaluated and included by the intermediate nodes.

In extended AODV, each node computes $\mathrm{BW}_{\text {local }}$ and $\mathrm{BW}_{\mathrm{c} \text {-neigh }}$ as per (1) and (2) respectively. Also it calculates its residual lifetime probability (6). Every intermediate node, on receiving RREQ performs preliminary admission control as given in Fig.2. If the bandwidth requirement of the flow $\mathrm{BW}_{\text {flow }}$ is lower than node's local available bandwidth $\mathrm{BW}_{\text {local }}$ and contention neighborhood available bandwidth $\mathrm{BW}_{\mathrm{c}-\mathrm{neigh}}$, then it checks for link stability. Node's ID is added in the reverse path if $\mathrm{P}\left(\right.$ residual_lifetime $>\mathrm{T}_{\mathrm{r}}$ ) $* \mathrm{P}_{\mathrm{a}}>\mathrm{P}_{\mathrm{r} \text {-fail }}$. In case of failure, the RREQ is discarded. On success of the preliminary admission control the node sets up a reverse route entry in its routing table, adds its identifier in the RREQ packet. The value ' $\mathrm{P}_{\mathrm{a}}$ ', the accumulated survival probability is computed and updated in the RREQ packet. Then the node rebroadcasts the route request. Recording the sequence of hops in RREQ packet enables to determine the lower bound of the contention count of the complete route and also it can be used to eliminate circular routes.

When the intended destination receives a route request, it receives the full route and sends a route reply (RREP) back to the source along the same route. The destination may get different routes. 1. Each node computes $B W_{\text {local }}$ and $B W_{c-n e i g h}$ through passive listening.

2. On receiving RREQ packet, node extracts $B W_{\text {flow }}$ and $\left(P_{r_{-} \text {fail, }} T_{r}\right)$ parameters.

3. Each node predicts its $P\left(\right.$ residual_lifetime $\left.>T_{r}\right)$.

$$
\begin{aligned}
& \text { // checking for throughput } \\
& \text { if }\left(B W_{\text {local }}>B W_{\text {flow }}\right) \text { and }\left(B W_{c-n e i g h}>B W_{\text {flow }}\right) \text { then } \\
& / / \text { checking for link stability } \\
& \text { node reads ' } P_{a} \text { ', the product of survival } \\
& \text { probability of links; } \\
& \text { if }\left(P\left(\text { residual lifetime }>T_{r}\right) * P_{a}\right)<P_{r_{-} \text {fail }} \text { then } \\
& \text { node's ID is added in } R R E Q ;
\end{aligned}
$$

// $\mathrm{P}_{\mathrm{a}}$ value in the RREQ message is updated

$\mathrm{P}_{\mathrm{a}} \leftarrow \mathrm{P}_{\mathrm{a}}{ }^{*} \mathrm{P}$ (residual_lifetime $>\mathrm{T}_{\mathrm{r}}$ );

$\mathrm{RREQ}$ packet is rebroadcast;

else

// admission control fails

RREQ packet is discarded;

\section{Figure 2. Preliminary Admission Control Algorithm}

But all the routes partially satisfy the bandwidth requirement. Priority is given to the path involving less number of hops. The remaining routes are cached at the destination for certain time period in order to make use of them if final admission control fails for the first selected route. On receiving the RREP packet, a node performs final admission control as shown in Fig.3. A node needs its contention count information at this stage. Node's neighbour details are gathered through HELLO messages, contention neighbour details are obtained through passive listening to the medium. Using these details and the path information available in the RREP packet, a node calculates its actual contention count. Hence the node computes its actual bandwidth consumption of the flow ( $\left.\mathrm{BW}_{\mathrm{a}_{-} \text {flow }}\right)(5)$. If the actual bandwidth requirement of the flow $\left(\mathrm{BW}_{\mathrm{a} \_ \text {flow }}\right.$ is lower than node's local available bandwidth $\left(\mathrm{BW}_{\text {local }}\right)$ and contention neighbourhood available bandwidth $\left(\mathrm{BW}_{\mathrm{c}-\mathrm{neigh}}\right)$, final admission control succeeds otherwise it fails. On success of admission control, a soft reservation of bandwidth is made in the routing

\section{Node on the reverse path receives RREP packet.}

2. Node estimates its contention count $\left(\mathrm{C}_{\mathrm{ct}}\right)$ from path details.

3. Node computes its $B W_{a_{-} \text {flow }}$.

4. if $\left(\mathrm{BW}_{\text {local }}>\mathrm{BW}_{\mathrm{a}_{-} \text {flow }}\right)$ and $\left(\mathrm{BW}_{\mathrm{c}-\text { neigh }}>\mathrm{BW}_{\mathrm{a}_{\text {fllow }}}\right)$ then soft reservation for bandwidth requirement is made; RREP packet is forwarded to its predecessor node on the reverse path; else // admission control is successful
// admission control fails
RREP packet is discarded;

Figure 3. Final Admission Control Algorithm 
table and RREP is forwarded to its immediate predecessor. On failure of final admission control, an admission failure message is sent to the destination via the same reverse route. Route error message (RERR) of AODV is modified in our approach to carry admission failure message on the reverse route. It enables cancellation of bandwidth reservation by the successor nodes. On receiving the admission failure message, the destination selects another fresh cached route and sends a RREP. On successfully receiving RREP, a source has enough end-to-end bandwidth reserved on entire route. Also route is made up of sufficiently strong links. Communication can start at each node on the path, bandwidth reservation is refreshed by the arrival of data packets. The bandwidth reservation at the node automatically expires, if no data packet arrives due to link breakage.

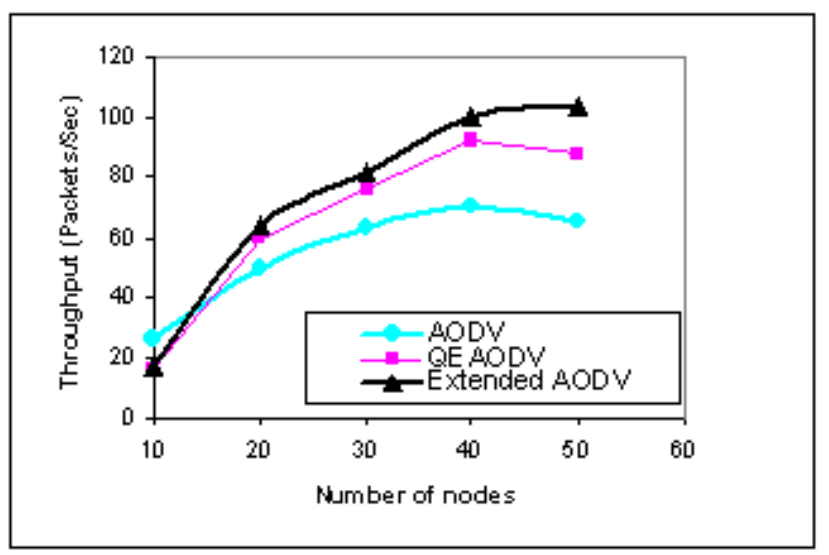

Figure 4. Throughput of Extended AODV.

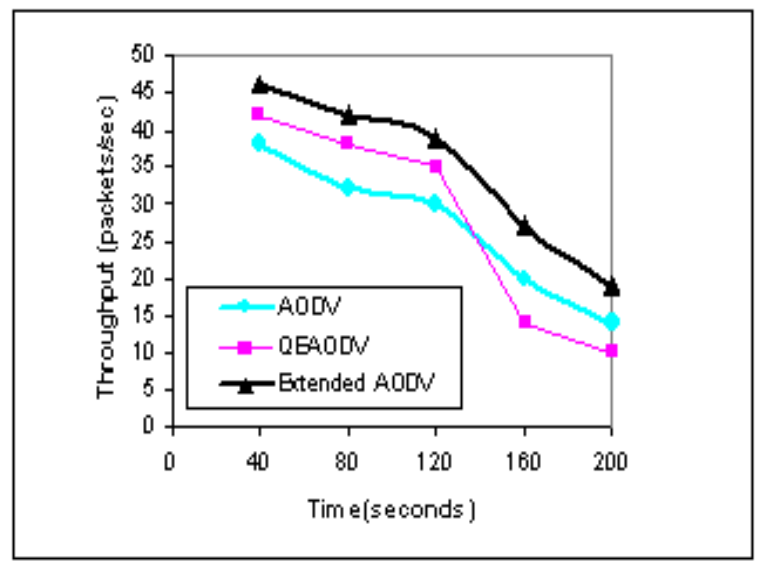

Figure 5. Throughput for different simulation times.

\section{SIMULATION AND RESULTS}

The extended multi constraint routing protocol is implemented using the NS-2 network simulator [16] by modifying the code of AODV protocol. AODV protocol already exists in the network layer. A modification is done in the MAC layer to capture the signal strength. In extended AODV the packet structure of RREQ

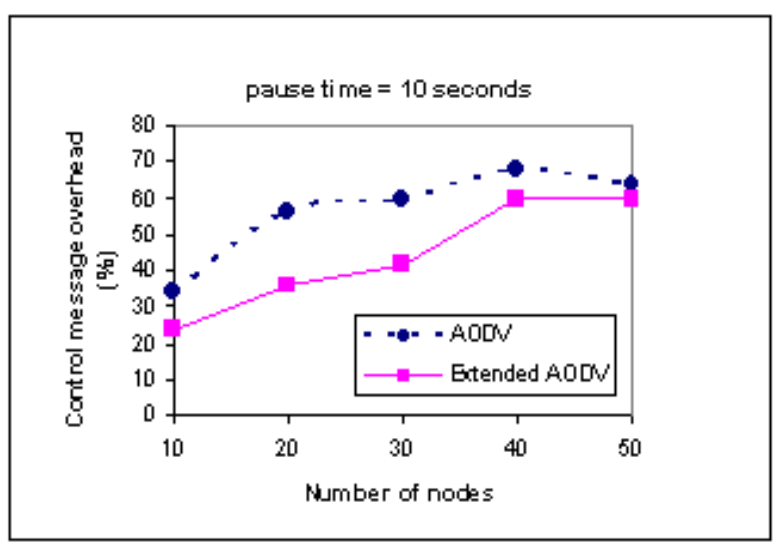

Figure 6. Control message overhead for pause time $=10$ sec

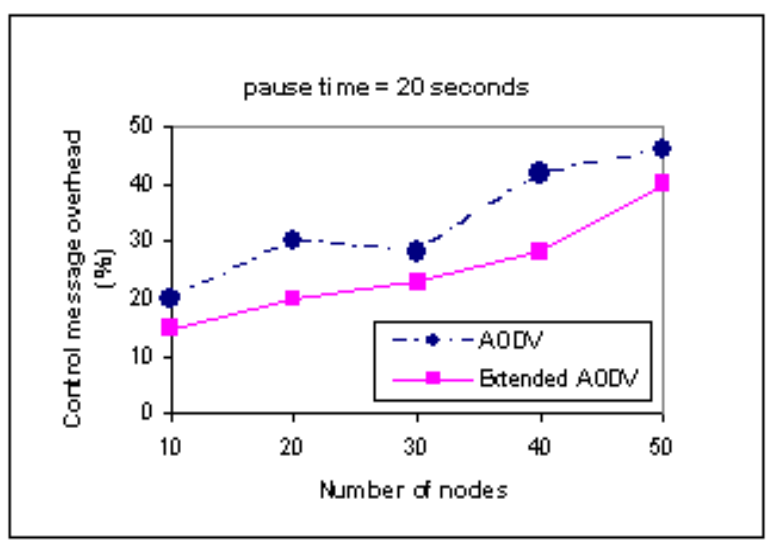

Figure 7. Control message overhead for pause time $=20 \mathrm{sec}$

is changed to carry required metrics information. The routing table structure is also changed to hold the additional details.

Simulations are run for different scenarios. Different scenarios are created using 10, 20, 30, 40 and 50 nodes. Protocol evaluations are based on the simulation of wireless nodes forming an ad hoc network, moving about over a rectangle. Rectangle size is $1000 \mathrm{~m}$ $\mathrm{x} 1000 \mathrm{~m}$, simulation time is 200 seconds. At medium access control (MAC) layer the 802.11 protocol is used. Radio transmission range of a node is set to $200 \mathrm{~m}$ and the carrier sensing range is set $400 \mathrm{~m}$. Each flow generated 10 packets per second. Each packet size is 512 bytes. The bandwidth of the channel is 2 Mbps. A number of simultaneous CBR flows are made. Speed of nodes is varied from $2 \mathrm{~m} / \mathrm{s}$ to $10 \mathrm{~m} / \mathrm{s}$. The performance of the extended AODV is compared with normal AODV and also with QEAODV [10] which is designed based on bandwidth constraint alone in terms of throughput, overhead and network performance ratio. Throughput of extended AODV gets increased significantly when compared with normal AODV and single constraint based QoS routing(QEAODV) as shown in Fig. 4. In Fig. 5 throughput 
of QEAODV is high till the average simulation time of 130 seconds, beyond that throughput gets decreased. This is due to the effect of nodes mobility. But extended AODV shows the consistent improvement in throughput for the entire simulation time. From Fig. 6 and Fig. 7, it is inferred that control message overhead of extended AODV execution is comparatively lower than normal AODV execution. Overhead incurred by extended AODV is almost similar to QEAODV. Also as the nodes' pause time value increases, control message overhead decreases drastically.

\section{CONCLUSION}

In this paper we presented multi constraint based routing technique to incorporate Quality of Service into routing. The concept of available bandwidth estimation of a node is performed by accounting contention neighbours bandwidth utilisation. Admission control process for a new flow involves two phases, so that reliable path can be achieved at the end. The existing AODV performs routing with low control overhead and effective packet transmission. But do not have QoS support. We extended the normal AODV to perform path finding that meets the application stipulated bandwidth requirement and link stability metrics. Our path finding approach is modified in such a way that it deals with common medium sharing problem of the adhoc networks effectively. Inclusion of link stability metric ensures sustained QoS support till the complete data transmission. The extended AODV performs path finding with less overhead by adopting passive approach of listening to the medium. Simulations show that contention aware, link stability based route finding technique performs better than AODV in terms of throughput and control message overhead. It improves packet delivery ratio greatly without affecting the overall end-to-end throughput of existing flows.

\section{REFERENCES}

[1] Z. Fan, "QoS routing using lower layer information in adhoc networks," in Proc. Personal, Indoor and Mobile Radio Communications Conf., pp. 135-139, Sep. 2004.

[2] Y. Yang and R. Kravets, "Contention-aware admission control for ad hoc networks," IEEE Trans. Mobile Comput., vol. 4, pp. 363-377, Aug 2005.

[3] L. Hanzo and R. Tafazolli, " A Survey of QoS Routing Solutions for Mobile Ad Hoc Networks,"IEEE Communications Surveys, vol. 9, No. 2, 2nd Quarter, 2007.
[4] C. E. Perkins and E. M. Royer and S. R. Das, "Ad Hoc on Demand Distance Vector (AODV) Routing," Internet Draft, draft-ietf-manet-aodv-08.txt, 2001.

[5] Kishor S. Trivedi, "Probability and Statistics with Reliability, Queueing and Computer Science Applications," PHI, 2005.

[6] C. Perkins and P. Bhagwat, "Highly dynamic destination sequenced distance vector routing (DSDV) for mobile computers," in Proc. ACM SIGCOMM'94, pp. 234-244.

[7] I. Rubin and Y.C. Liu, "Link stability models for QoS ad hoc routing algorithms," in Proc. 58th IEEE Vehicular Technology Conf., Vol. 5, pp. 3084-3088, Oct. 2003.

[8] S. Singh, M. Woo, and C.S. Raghavendra, "Power-aware routing in mobile ad hoc networks," in Proc. Int. Conf. Mobile Computing and Networking, pp. 181-190, 1998.

[9] F. Kuipers and P. VanMieghem, "Conditions that impact the complexity of QoS routing," IEEE/ACM Trans. Networking, vol 13, no. 4, pp. 717-730, 2005.

[10] D.S. Thenmozhi and R. Lakshmipathi, "Quality of Service Enhanced Routing in Mobile Ad hoc Networks," IJCSIS, Vol 8, No. 5, pp. 34-40, 2010.

[11] S. Murthy and J. J. Garcia-Luna-Aceves, "An efficient routing protocol for wireless networks," ACM Mobile Networks Applicat. J., Special Issue on Routing in Mobile Communication Networks, 1996.

[12] V. D. Park and M. S. Corson, "A Highly adaptive distributed routing algorithm for mobile wireless networks," in Proc. IEEE INFOCOM'97, pp. 1405-1413.

[13] D. Johnson and D. Maltz, "Dynamic source routing in ad hoc wirelesss networks," Mobile Computing. E. Imielinski and H. Korth, Eds. Norwell, MA. Kluwer, 1996.

[14] C. K. Toh, "Associativity-based routing for ad hoc mobile networks," Wireless Personal Commun., vol. 4, pp. 103-139,1997.

[15] Z. J. Haas and M. R. Pearlman, "The performance of query control schemes for the zone routing protocol," in Proc. IEEE Symp. Computers and Communication, 1998.

[16] S. McCanne, S. Floyd, S. Fall, K. Varadhan: The Network Simulator NS2 (1995). The VINT Project, available at http://www.isi.edu/nsnam/ns. 